\title{
De la acumulación de capacidades tecnológicas a la planificación geoestacionaria en la Argentina
}

| Fecha de recibido: 10 de agosto del 2020 | Fecha de aprobación: 25 de septiembre del 2020 |

Paulo Daniel Pascuini

Magíster en Economía

Universidad de Buenos Aires Rol de investigador: teórico, experimental y escritura https://orcid.org/0000-0002-6953-9819

$\triangle$ paulo.pascuini@fce.uba.ar

Cómo citar este artículo: Pascuini, P. D. (2020). De la acumulación de capacidades tecnológicas a la planificación geoestacionaria en la Argentina. Revista Ciencia y Poder Aéreo, 15(2), 53-67. https://doi.org/10.18667/cienciaypoderaereo.676 


\section{De la acumulación de capacidades tecnológicas a la planificación geoestacionaria en la Argentina}

\section{From Technological Capabilities Accumulation to Geostationary Planification in Argentina}

Resumen: en los últimos 25 años, la Argentina alcanzó una relevante serie de hitos tecnológicos al diseñar y construir satélites propios, entre ellos, cuatro en cooperación con la NASA, dos que llevan la antena de radar más grande puesta en un satélite con fines civiles, y dos geoestacionarios de telecomunicaciones. En el 2015 el Congreso de la Nación Argentina promulgó una ley que aprueba y declara de interés nacional el "Plan Satelital Geoestacionario Argentino 2015-2035", el cual incluye un plan de construcción de satélites geoestacionarios de telecomunicaciones. El objetivo de este trabajo es analizar en qué medida la ley aprobada por el Congreso posibilita la preservación de las capacidades tecnológicas acumuladas en materia espacial, o tiene capacidad para hacerlo a futuro. La metodología empleada para alcanzar dicho objetivo consistió en una revisión de la literatura existente sobre la industria satelital argentina, entrevistas semiestructuradas a referentes del ecosistema en distintas ciudades de la Argentina, y el estudio de la ley y el resto de la normativa vigente. A partir del análisis de aspectos técnicos y económicos de la ley, y de los desvíos existentes a julio del 2020 respecto de la cronología de construcción de satélites programada en su anexo, este trabajo encuentra que su incumplimiento es un factor que pone en peligro recursos muy valiosos, como son las posiciones orbitales geoestacionarias, o las capacidades tecnológicas acumuladas sobre la base de los desarrollos nucleares iniciados a finales de la década del 40 . Así mismo, se encontró que la ley carece de criterios que incorporen la dinámica tecnológica y económica fluctuante del sector. A modo de conclusión, se discuten las implicaciones de la ley para el sector y, se propone una serie de elementos a considerar en la actualización prevista para el plan de construcción de satélites.

Palabras clave: Argentina; industria aeroespacial; satélites artificiales; comunicación satelital.

Abstract: During the last 25 years, Argentina has reached a relevant series of technological milestones by designing and building its own satellites, including four in cooperation with the NASA, two that carry the largest radar antenna placed on a satellite for civil purposes, and two geostationary telecommunications satellites. In 2015, the Congress of the Argentine Nation enacted a law that approved and declared the "Argentine Geostationary Satellite Plan 2015-2035" as a matter of national interest. The Law includes a plan for the construction of geostationary telecommunications satellites. The objective of this paper is to analyze to what extent the Law approved by the Congress enables the preservation of accumulated technological capabilities in the space sector or has the capacity to do so in the future. The methodology used to achieve this objective consisted of a review of the existing literature on the Argentine satellite industry, semi-structured interviews with referents of the ecosystem in different cities of Argentina, and the study of the law and current regulations. Based on the analysis of the technical and economic aspects of the law, and the existing deviations as of July 2020 with respect to the satellite construction chronology programmed in its annex, this paper finds that non-compliance is a factor that endangers highly valuable resources such as geostationary orbital positions or the technological capabilities accumulated on the basis of the nuclear developments initiated in the late 1940s. In addition, it was found that the law lacks criteria that incorporate the fluctuating technological and economic dynamics of the sector. To conclude, the paper discusses the implications of this law for the sector and proposes a series of elements to consider in the prearranged updating of the satellite construction plan.

Keywords: Argentina; aerospace industry; artificial satellites; communication satellites. 


\section{Da acumulação de capacidades tecnológicas ao planejamento geoestacionário na Argentina}

Resumo: Nos últimos 25 anos, a Argentina atingiu uma série relevante de marcos tecnológicos desenhando e construindo seus próprios satélites, incluindo quatro em cooperação com a NASA, dois carregando a maior antena de radar colocada em um satélite para fins civis e dois geoestacionários de telecomunicações. Em 2015, o Congresso da Argentina promulgou uma lei que aprova e declara de interesse nacional o "Plano de Satélites Geoestacionários Argentinos 2015-2035" incluindo um plano para a construção de satélites de telecomunicações geoestacionários. $O$ objetivo deste trabalho é analisar em que medida a lei aprovada pelo Congresso possibilita a preservação das capacidades tecnológicas acumuladas em matéria espacial ou tem capacidade para fazê-lo no futuro. A metodologia usada para atingir este objetivo consistiu em uma revisão da literatura existente sobre a indústria argentina de satélites, entrevistas semiestruturadas com referentes do ecossistema em diferentes cidades da Argentina, e o estudo da lei e das normas vigentes. Com base na análise dos aspectos técnicos e econômicos da lei, e dos desvios existentes respeito à cronologia de construção de satélites programada em seu anexo, este trabalho confirma que a falta de cumprimento é um fator que compromete muitos recursos como as posições orbitais geoestacionárias, ou as capacidades tecnológicas acumuladas baseadas nos desenvolvimentos nucleares iniciados no final da década de 1940. Por sua vez, constatou-se que a lei carece de critérios que incorporem as flutuantes dinâmicas tecnológicas e econômicas do setor. Finalmente, o artigo aborda as implicações da lei para o setor e propõe uma série de elementos a serem considerados na atualização planejada do plano de construção do satélite.

Palavras-chave: Argentina; Indústria aeroespacial; Satélites artificiais; Comunicação por satélite. 


\section{Motivación y objetivo}

Un satélite artificial es un dispositivo tecnológico que orbita alrededor del planeta (Gallicchio, 2017), y aunque algunos de ellos se utilizan con fines militares, la mayoría desarrollan varias actividades de carácter civil. Dentro de estas se pueden encontrar satélites de telecomunicaciones, los cuales proveen servicios de televisión, telefonía y transmisión de datos; de posicionamiento, que brindan servicios de posicionamiento global; de observación terrestre y meteorológicos, cuya finalidad es analizar los patrones climáticos como también observar la vegetación de los bosques, cauces de los ríos, entre otros; y los científicos, empleados para la investigación y ampliación de conocimientos respecto a los recursos en la tierra y el ámbito espacial. La infraestructura de los satélites se puede dividir en dos partes: la carga útil (payload) y el bus. La carga útil son todos los objetos necesarios para cumplir la misión del satélite, los cuales dependen del objetivo de la misión y pueden ser cámaras, telescopios, entre otros. Los demás subsistemas que componen el satélite, como por ejemplo, el sistema de propulsión, control térmico, control de estabilidad, y de energía, son los componentes que permiten que el satélite se mantenga unido y en funcionamiento, los cuales conforman el bus (Solis-Santomé \& Santos-Reyes, 2016).

Existen diversas clasificaciones para los recorridos y posicionamientos de los satélites alrededor de la Tierra (Urbina-Carrero, 2017). No obstante, la clasificación de mayor referencia es la de altura, medida desde la superficie terrestre y en la que se posicionan los satélites en el espacio, a saber: Órbita Terrestre Baja (Low Earth Orbit, LEO), Órbita Terrestre Media (Medium Earth Orbit, MEO) y Órbita Geosincrónica (Geosynchronous Orbit, GSO). Un caso particular de los Gso son los satélites de Órbita Geoestacionaria (Geostationary Orbit, GEO). Esta órbita es la más utilizada por los satélites de telecomunicaciones (a los que en adelante, se hará referencia como satélites GEO), que se sitúan en posiciones orbitales geoestacionarias (POG), cuya asignación a nivel internacional está administrada por la Unión Internacional de Telecomunicaciones (UIT). Respecto de los satélites de telecomunicaciones, recientemente se han desplegado grandes constelaciones de estos en LEO, como Starlink o OneWeb (Xu \& Zhang, 2018; Orvola et al., 2020). Sin embargo, el futuro de las constelaciones de satélites de telecomunicación fuera de la tradicional GEO, aún no está claro. Mientras que algunas pueden tener beneficios como la cobertura de zonas polares (Lee et al., 2016) o la complementariedad con dispositivos loT en zonas remotas (Qu et al., 2017; Chaari et al., 2019), también pueden acarrear varios problemas como el incremento en la posibilidad de colisión (Pardini \& Anselmo, 2020; 2019).

El sector satelital es uno de los pocos sectores industriales de alta tecnología en donde la Argentina ha generado capacidades de innovación propias y ha alcanzado un nivel relevante de reconocimiento internacional. En la historia reciente, esta acumulación de capacidades es resultado de un proceso a lo largo del cual el país fue capaz de diseñar y poner en órbita diferentes satélites, tanto de observación como de telecomunicaciones (López, Pascuini \& Ramos, 2017), y donde la creación de la cadena de valor en el sector ha requerido inversiones sostenidas durante más de 25 años. En este sentido, el caso argentino no se distingue de otras naciones donde, se ha verificado sistemáticamente que la creación de una cadena de valor requiere que estas inversiones se prolonguen por entre dos y tres décadas (Nagendra \& Basu, 2016). Sin embargo, un aspecto particular del caso argentino es que las capacidades tecnológicas, que fueron las bases para los proyectos satelitales en la década del 90 , se acumularon previamente en el sector nuclear ${ }^{1}$.

El importante desarrollo del sector espacial argentino se da en el marco de un conjunto de normas que afectan tanto las posibilidades de desarrollo tecnológico, como la constitución o desarrollo de mercados y actores en el resto de la cadena de valor. Recientemente, han emergido en la agenda de gestión de la política de comunicaciones y de ciencia y

1 Resulta interesante que tal como las capacidades en el área nuclear propiciaron el desarrollo en el área satelital, las capacidades acumuladas en esta última propiciaron el desarrollo en el área de radares. Ver la tercera sección de este trabajo. 
tecnología de la Argentina una serie de elementos como la tercera revisión del plan espacial "Argentina en el espacio 2016-2027”, elaborado por la Comisión Nacional de Actividades Espaciales (CONAE); el proyecto para actualizar el Reglamento de Gestión y Servicios Satelitales del año 1999; o la Ley de Desarrollo de la Industria Satelital (Ley n. ${ }^{\circ}$ 27.208) que, promulgada por el Congreso de la Nación el 9 de noviembre del 2015, aprueba y declara de interés nacional el "Plan Satelital Geoestacionario Argentino 2015-2035”.

De acuerdo con la hipótesis de que las definiciones e indefiniciones técnicas, y viabilidad económica de los aspectos incluidos en la ley, además de los desvíos existentes a julio del 2020 respecto de la cronología de construcción de satélites, condicionan en distinta medida y sentido la preservación y el desarrollo de las capacidades tecnológicas, este trabajo se propone contribuir a que futuras decisiones de política resulten en una profundización de este largo proceso de acumulación de capacidades. Más aun, dado que la CONAE no incluye satélites de telecomunicaciones en el Plan Espacial Nacional, las demoras en el plan de construcción de satélites geoestacionarios de telecomunicaciones contenido en esta ley, ponen en riesgo la preservación de las POG asignadas por la UIT, las cuales constituyen un recurso escaso (Jakhu, 2007). Por estos motivos, el país se encuentra en un punto de inflexión en la toma de decisiones de políticas públicas que puedan afectar el desarrollo de su industria espacial.

El objetivo particular de este trabajo consiste en analizar en qué medida la Ley de Desarrollo de la Industria Satelital, posibilita la preservación de las capacidades tecnológicas acumuladas en materia espacial, o tiene capacidad para hacerlo a futuro. El artículo se organiza de la siguiente manera: en la segunda sección se presentará el diseño metodológico y las fuentes de información consultadas para atender el objetivo del trabajo. En la tercera sección, se hará una reseña sobre la acumulación de capacidades tecnológicas desde fines de la década del 40, que permitieron a la Argentina alcanzar una serie de hitos relevantes en el área satelital, a la par de los hechos más relevantes a nivel institucional que la acompañaron. En la cuarta sección se realiza un análisis sobre los aspectos de la Ley de
Desarrollo de la Industria Satelital (27.208), en particular, el plan de construcción de satélites, que promueven o inhiben la preservación y el desarrollo de las capacidades tecnológicas reseñadas en la sección anterior. Por último, en la quinta sección se discuten los resultados del análisis realizado y se presentan las principales conclusiones de este trabajo.

\section{Metodología y fuentes de información}

Para atender el objetivo particular del trabajo, se diseñó un estudio de investigación cualitativo que permitiera evaluar las reglas de juego en las que se enmarca la fabricación de satélites en la Argentina. En primer lugar, se revisó la literatura existente sobre la industria satelital para la Argentina, incluyendo lo que refiere a la acumulación de capacidades en esta área tecnológica, y las leyes, normativas y proyectos vigentes en el ámbito espacial. En segundo lugar, se realizó un trabajo de campo basado en entrevistas semiestructuradas de carácter exploratorio llevadas adelante en (i) la Ciudad Autónoma de Buenos Aires; (ii) la ciudad de La Plata, provincia de Buenos Aires; y (iii) la ciudad de San Carlos de Bariloche, provincia de Río Negro. Durante estas entrevistas se consultaron referentes y especialistas en los distintos eslabones de la cadena de valor, desde la fabricación de satélites y la provisión de capacidad satelital, hasta la prestación de servicios satelitales, que sirvieron para contribuir a la contextualización de la política y de los diferentes hitos en la normativa, y para dar sentido a la información técnica de los distintos marcos normativos estudiados tanto en los aspectos tecnológicos como legales.

Los elementos y dimensiones presentados en este estudio surgen de diferentes fuentes de información. El análisis de la normativa nace de la revisión de la Ley de Desarrollo de la Industria Satelital (Ley n. ${ }^{\circ}$ 27.208) y su anexo, también de entrevistas con (i) directivos y exdirectivos de los principales actores de la economía del espacio de la Argentina, y (ii) expertos legales en el área. 


\section{Una reseña sobre la acumulación de capacidades tecnológicas e institucionales}

El inicio de la acumulación de capacidades en el área nuclear se remonta al fin de la Segunda Guerra Mundial, que trajo consigo una importante corriente migratoria de científicos alemanes hacia la Argentina. Entre ellos se encontraba Ronald Richter, quien presentó al presidente Perón una propuesta para fabricar un reactor nuclear, cuyo desarrollo comenzó en la provincia argentina de Córdoba y siguió en la isla Huemul, ubicada en el lago Nahuel Huapi en el sur argentino. Para darle un marco administrativo al proyecto, denominado Proyecto Huemul, en 1950 se creó la Comisión Nacional de Energía Atómica (CNEA), empero, el proyecto fue cancelado debido a que carecía de fundamento científico. Como consecuencia, la CNEA decidió aprovechar las instalaciones e investigaciones ya comenzadas con el fin de continuar desarrollando la física nuclear, para lo cual fundó un instituto que poco después se convertiría en el Instituto Balseiro, el cual, actualmente es un centro académico reconocido en el ámbito internacional en los campos de la física experimental y la ingeniería nuclear. Egresados de este fundaron en 1972 la firma Investigaciones Aplicadas, una entidad que la CONAE, en convenio con la provincia de Río Negro, constituiría en 1976 como la empresa INVAP S.E. ${ }^{2}$ (en adelante, INVAP). Esta empresa inicialmente se dedicó al sector nuclear, en el cual diseña y provee sistemas para reactores nucleares, pero también vende, llave en mano, reactores para investigación y fabricación masiva de radioisótopos de uso médico (López, Pascuini \& Ramos, 2019). La acumulación de capacidades, le ha permitido a INVAP diversificar su cartera de productos (Quiroga, 2020), y hoy, se destaca en el área nuclear, satelital y radar.

En 1991, debido a las presiones internacionales, se canceló un proyecto misilístico denominado Cóndor

2 S.E. es en la Argentina una figura societaria correspondiente a Sociedad del Estado.
II, que había tenido sus inicios a fines de la década del 70 y que llevaba adelante la Fuerza Aérea Argentina. A partir de su cancelación, se creó la CONAE, un organismo civil que heredó parte del personal civil vinculado al proyecto cancelado y las instalaciones aeroespaciales de la Fuerza Aérea Argentina, ubicadas en la Ciudad de Buenos Aires y en otras tres provincias:

La CONAE se ocupa de diseñar y ejecutar el Plan Nacional Espacial, cuya primera versión entró en vigencia en 1995, y si bien en estos planes se especifican los objetivos clave para los proyectos satelitales, de lanzadores y de segmentos terrenos en el corto, mediano y largo plazo, una carencia es que no abarcan el área de telecomunicaciones (López, Pascuini \& Ramos, 2019, p. 120).

El hecho de que el Plan Nacional Espacial no abarque el área de telecomunicaciones no es menor, ya que los ingresos allí son mucho mayores que en otros segmentos. Los ingresos globales generados para el 2018 por la industria satelital fueron cercanos a los USD 277,8 mil millones (MM), que se distribuyen en aproximadamente USD 128,5 MM referidos a los servicios satelitales, USD 125,2 MM correspondientes a equipamiento terrestre, USD 19,5 MM a construcción de satélites, y USD 6,2 MM correspondientes a servicios de lanzamiento. Es así que el segmento de negocio que más ingresos genera en la industria es el de servicios satelitales, dentro de los cuales los de telecomunicaciones son los que llevan la mayor proporción. A saber, la distribución de ingresos dentro de los servicios satelitales es: USD 94,2 MM en televisión, USD 17,9 en servicios fijos, USD 5,8 MM en radio, USD 4,1 MM en móviles, USD 2,4 MM en banda ancha, y por último, USD 2,1 MM en imágenes ${ }^{3}$.

En 1989, previo a la creación de la CONAE, se realizó un acuerdo de cooperación tecnológica entre Estados Unidos y la Argentina, seguido por una gestión bilateral entre la NASA y la, en esos entonces, Secretaría

3 Información de la Satellite Industry Association (SIA), puede encontrar la referencia de consulta en el listado final. 
de Ciencia y Tecnología de Argentina para llevar adelante la serie de satélites SAC (cuatro satélites lanzados entre 1996 y el 2011). En ese momento la única empresa en el país que podría participar de esa iniciativa era INVAP que, si bien hasta ese momento no había trabajado en esta industria, había acumulado capacidades tecnológicas compartidas entre el área satelital y nuclear. Según López, Pascuini \& Ramos (2018), estas capacidades incluyen electrónica (diseños de circuitos electrónicos, producción y testeo), sistemas de monitores y control, análisis estructural de objetos físicos (e.g. resistencia a la vibración), análisis térmico y químico, garantía de calidad, desarrollo de software y mecanizado especial de componentes de alta complejidad. A estas capacidades se les suma la habilidad para pronosticar los efectos de la radiación, ya que así como es necesario estimar los daños potenciales causados por la radiación nuclear en los componentes de un reactor, también lo es pronosticar los daños potenciales causados por la radiación cósmica en los componentes de un satélite.

El progreso tecnológico en materia espacial puede medirse de diversas maneras. Una de ellas es a través de la Space Technology Ladder (STL), la cual consiste en una serie de hitos en el desarrollo espacial ordenados según su complejidad técnica, que a su vez, en el caso de los hitos satelitales, se subdividen en niveles de acuerdo con el grado de autonomía tecnológica local alcanzada (Wood \& Weigel, 2012). En un trabajo reciente López, Pascuini \& Ramos, (2018) aplican la STL para el caso argentino y encuentran que este país ha experimentado significativos avances en el desarrollo tecnológico de las actividades espaciales, entre los que se destacan la posesión y operación de satélites de observación de la Tierra en LEo desarrollados y fabricados en el marco de colaboraciones internacionales, y de satélites GEO de telecomunicaciones desarrollados y fabricados localmente 4 .

El primer lanzamiento de un satélite con fines científicos (SAC-B) ocurrió en 1996 y estuvo a cargo de

4 Hito que fue alcanzado por primera vez en el 2014 con la puesta en órbita del ARSAT 1. la CONAE, con INVAP como principal contratista, que al momento ha concluido otras 3 misiones satelitales: SAC-A, C y D, todas ellas en cooperación con la NASA. En octubre del 2018 se lanzó el satélite de observación de la Tierra SAOCOM 1A y en agosto del 2020 el SAOсом 1B, ambos satélites llevan la antena de radar más grande puesta en un satélite en órbita con fines civiles y fueron desarrollados también por la CONAE e INVAP. El desarrollo de estos satélites de observación radar con microondas en banda L, conforman un hito que solo Japón había alcanzado hasta el momento. De hecho, INVAP en la última década ha sido capaz de producir radares primarios, secundarios y meteorológicos a partir de las capacidades acumuladas en relación al instrumento radar de los satélites SAOCOM. Quiroga y Aguiar (2016) explican que, si bien para construir radares INVAP contrató ingenieros especializados en radio frecuencias, sistemas de detección y comunicaciones y procesamiento de señales, la generación previa de instrumentos de radares, específicamente los desarrollos de los componentes de la antena SAR (por la sigla en inglés de Synthetic Aperture Radar) para el satélite SAOCOM (e.g. el generador de señales y sincronismos y el módulo transmisor/receptor), sirvieron para adquirir conocimientos que posteriormente agilizaron la fabricación, por ejemplo, de radares secundarios.

Por otra parte, en el 2006 se creó la Empresa Argentina de Soluciones Satelitales S.A. (en adelante, ARSAT), que en la actualidad depende de la Secretaría de Innovación Pública, dependiente de la Jefatura de Gabinete, y del Ministerio de Economía. Esta empresa fue constituida con el objetivo de diseñar, desarrollar, lanzar y explotar comercialmente satélites geoestacionarios de telecomunicaciones. Si bien la fabricación y el lanzamiento de satélites están comprendidos dentro de sus objetivos, ARSAT pertenece principalmente al eslabón de operadores dentro de la cadena de valor del sector, proveyendo capacidad satelital a sus clientes, quienes la transforman en servicios que satisfacen necesidades de telecomunicación. La empresa participó, en conjunto con INVAP en el desarrollo de los satélites geoestacionarios de telecomunicaciones ARSAT 1 y 2 , que se encuentran actualmente en órbita. Esto situó a la Argentina dentro del reducido 
grupo de ocho actores ${ }^{5}$ con capacidades suficientes para construir localmente satélites geoestacionarios de telecomunicaciones ${ }^{6}$.

\section{El plan de construcción de satélites argentinos 2015-2035}

Esta sección se propone analizar los principales aspectos tanto técnicos como económicos del plan de construcción de satélites argentinos 2015-2035, en el contexto de la Ley de Desarrollo de la Industria Satelital. Puntualmente se estudian sus limitaciones, que surgen de definiciones e indefiniciones técnicas, su viabilidad económica con base en el plan de negocios que lo respalda, y los desvíos existentes a julio del 2020 respecto de la cronología de construcción de satélites y sus implicaciones. En estas dimensiones de análisis se sugiere una serie de elementos que podrían ser de suma relevancia en una posible actualización de dicho plan.

La Ley de Desarrollo de la Industria Satelital (27.208), promulgada por el Congreso de la Nación el 9 de noviembre del 2015, aprueba y declara de interés nacional el "Plan Satelital Geoestacionario Argentino 2015-2035" . Aunque en esta se establece que el Poder Ejecutivo Nacional, por intermedio de ARSAT, es quien ejecutará las acciones necesarias para cumplir el Plan Satelital y actualizarlo mediante revisiones cada tres años donde se propongan las modificaciones necesarias, a julio del 2020 no se ha realizado y hecho pública la revisión del plan, es decir, habiendo transcurrido

5 A saber: Argentina, China, Estados Unidos, India, Israel, Japón, Rusia, y la Unión Europea. Se espera que este grupo de actores se amplíe próximamente, en la medida que otros países avanzan en la curva de aprendizaje tecnológico. Por ejemplo, se proyecta que Turquía lance en el 2022 su satélite GEO Türksat-6A construido localmente.

6 Para más detalles sobre los principales actores y la organización institucional del sector en la Argentina véase López, Pascuini \& Ramos (2017), para más información sobre el rol de la política pública en la evolución del sector véase López, Pascuini \& Ramos (2019), y para datos sobre la evolución de capacidades tecnológicas consultar a López, Pascuini \& Ramos (2018).

7 Artículo 2 de la Ley de Desarrollo de la Industria Satelital (27.208). cuatro años y ocho meses de la promulgación de la ley. El Plan Satelital Geoestacionario Argentino 2015-2035 (que se incluye en un anexo dentro de la $\left(\right.$ ley $^{8}$ ), está compuesto por cuatro secciones y a su vez anexos (es decir, anexos del anexo de la ley), que amplían conceptos y elementos.

En los "objetivos" del plan se establece que, a la vez que se persigan mejoras de eficiencia, la esencia de estos es continuar y profundizar la actividad comercial y el desarrollo de nuevos servicios y plataformas satelitales. Ahora bien, respecto de la eficiencia de los satélites GEO de telecomunicaciones, un indicador de la misma es la relación entre cantidad de kilowatts de potencia del satélite por tonelada (López et al., 2017), y el plan informa que la tendencia mundial para el periodo de los años 1995 al 2020 marca una evolución de plataformas de 1,5 Watts por $\mathrm{kg}$ a 3 Watts por $\mathrm{kg}^{9}$. Sobre esto, el plan expone que la "Argentina, a través de ARSAT deberá alcanzar una plataforma de este tipo para 2020", lo cual se lograría, pasando de plataformas de propulsión química a plataformas con propulsión híbrida primero y después eléctrica, las cuales se denominan respectivamente ARSAT- $3 \mathrm{~K}^{10}$, ARSAT-3 $\mathrm{H}^{11}$ y ARSAT-3E ${ }^{12}$. No obstante, este objetivo no se alcanzará a tiempo siendo que a julio del 2020 ARSAT no ha concretado acciones, aunque sí ha manifestado intenciones para el desarrollo de un satélite que permita alcanzar esa eficiencia. Respecto a nuevas plataformas, se prevé la realización de actividades de investigación y se menciona la necesidad de incrementar el componente nacional de los satélites, lo que requiere análisis de costos y spillovers sobre otros sectores de la economía.

En el "Programa de gestión y vinculación institucional" se anuncia que para el desarrollo del plan se solicitaron ante la UIT nuevas asignaciones de frecuencia.

\footnotetext{
8 Se puede acceder al anexo de la ley desde el siguiente link: http:// servicios.infoleg.gob.ar/infolegInternet/anexos/250000-254999/254823/ ley27208.pdf

9 Aunque según información provista por especialistas del sector durante el trabajo de campo de esta investigación, podría haber en la actualidad plataformas con una mejor relación de Watts por kg.

10 Carga útil hasta $350 \mathrm{~kg}$ y potencia de $3,4 \mathrm{kw}$.

11 Carga útil hasta $600 \mathrm{~kg}$ y potencia de $7 \mathrm{kw}$.

12 De esta última, hay pocos datos disponibles en el anexo de la ley.
} 
Al momento de promulgarse la ley, los pedido ${ }^{13}$ eran cuatro: las extensiones de las POG $81^{\circ}$ Oeste y $71,8^{\circ}$ Oeste en banda Ka; y la $91,5^{\circ}$ Oeste y $38,5^{\circ}$ Oeste, ambas en bandas Ku y Ka. Una quinta solicitud vigente a julio del 2020 surge de la Advance Publication Information (API) enviada dos años después, en el 2017, para cubrir el riesgo de perder la $81^{\circ}$ Oeste en banda Ka. Sobre esto, ha resultado habitual que las POG de la Argentina corrieran riesgo de perderse. En varias ocasiones ha sido necesario cubrir transitoriamente una POG con satélites alquilados para preservarla, e incluso, en el 2004 se debió prorrogar la ocupación de la POG $81^{\circ}$ Oeste en bandas Ku y C. Esto presenta un riesgo en tanto desvíos en los plazos de construcción, que se detallarán más adelante, y que podrían conducir a la pérdida de una Pog. Tal es el caso de las POG $71^{\circ}$ Oeste en banda Ka, $38,5^{\circ}$ Oeste y $91,5^{\circ}$ Oeste en bandas Ku y Ka, cuyo vencimiento tiene fecha en el 2021, siendo que a julio del 2020 aún no se han publicado planes concretos para su ocupación. En caso de que se pretendiera ocupar alguna de ellas con un satélite fabricado localmente, dicha construcción debería contemplar alguna versión de plataforma con menores tiempos de construcción que la del ARSAT 1 y 2, ya que el plazo mínimo para fabricar un satélite de este tipo puede llegar a los 30 meses $^{14}$ (López \& Pascuini, 2018). De considerarse alguna alternativa tecnológica deberán contemplarse también los tiempos de desarrollo de la plataforma. Además, se evidencia la subutilización de un recurso valioso y escaso como las POG. Si alguna posición no pudiera ser ocupada por un satélite fabricado localmente antes de su vencimiento, se podría obtener rentabilidad mediante la subasta o concurso de la POG, operando un satélite, llegando al fin de su vida útil, alquilado a un operador satelital hasta que se disponga de un satélite propio, o asociándose con un tercero que aporte el satélite como contrapartida de

13 Para más información sobre el proceso de solicitud y asignación de POG por parte de la UIT, consulte el Reglamento de radiocomunicaciones, referenciado en el listado final.

14 De hecho, en una carta del Subsecretario de Planeamiento del ex Ministerio de Comunicaciones al Secretario de Tecnologías de la Información y las Comunicaciones del 28 de marzo del 2017, se afirma que la construcción del ARSAT 3 "requiere un plazo de 2 a 5 años" (Miguel, 2017, p. 2). la utilización de la POG argentina, compartiendo la capacidad satelital (Hosted Payload model).

Así mismo, el "Programa de desarrollo de servicios de contenidos satelitales" señala que el mercado de contenidos es el que mayores ingresos genera, en comparación con el de construcción y lanzamiento de satélites, en el plan se enumeran y detallan nichos importantes del mercado de contenidos, a saber: (i) redes corporativas y de gobierno; (ii) provisión de servicios de banda ancha satelital (HTS ${ }^{15}$ ); (iii) distribución de señales de TV a cable-operadores del país y la región; y (iv) distribución de televisión directa al hogar.

El "Programa de desarrollo de plataformas satelitales" hace referencia al rol del Estado en el proceso de innovación a través de un mejor acceso al capital y de la asunción de riesgos mayores y plazos de retorno más largos que los actores privados. Se argumenta que las inversiones asociadas al desarrollo de la industria espacial generan externalidades en otras industrias que deben considerarse desde la perspectiva del Estado, e incluirse en la decisión Make or Buy nacional o importado según costos y niveles de complejidad necesarios para su producción local. El desarrollo de materiales y software, son dos ejemplos de tareas que permitirían generar desarrollos útiles para otras industrias.

El "Programa de fabricación de satélites" especifica la construcción de ocho unidades. Mientras que dos se destinarían a la venta, los otros seis incluyen: dos para los reemplazos de los ARSAT 1 y 2; dos para las POG 81 y 71,8 en banda Ka; y dos para las POG en trámite ${ }^{16}$. Respecto de las plataformas de estos ocho satélites: (i) el ARSAT 3 utilizaría la plataforma ARSAT-3K (la misma que los ARSAT 1 y 2) y prestaría servicios en banda Ka y Ku; (ii-vi) si bien cada satélite tiene asignado con qué plataforma va a funcionar, el plan aclara: "Los cinco siguientes tendrán una plataforma química, híbrida

15 Acrónimo para High Throughput Satellite, tecnología que según el diseño puede hasta quintuplicar la relación $\mathrm{MHz} /$ Mbit de los satélites tradicionales.

16 Respecto a esas dos Pog ya solicitadas, la ley las menciona como A y $B$, y dice que están en trámite. Revisando las solicitudes vigentes a ese momento, surge que estas serían la POG $91,5^{\circ}$ Oeste y $38,5^{\circ}$ Oeste solicitadas en bandas Ku y Ka. 
o eléctrica según oportunidad y conveniencia"; (vii-viii) para los remplazos de los ARSAT 1 y 2 se estipula que se va a dar inicio a la construcción de dos plataformas eléctricas, sin dar mayores detalles sobre estas.

El cronograma estimado de fabricación de satélites va desde el 2015 al 2035, se prevé entre tres o cuatro años de construcción dependiendo del satélite y, a excepción del ARSAT 3, ninguno tiene nombre asignado, están enumerados según la plataforma. No se plantean especificaciones respecto de la pisada que tendrán aquellos satélites que aportarían ingresos por venta de servicios, solo dando lugar a las especulaciones propias en los casos en los cuales vayan a ocupar las POG asignadas hasta la actualidad a la Argentina.

A continuación, en la tabla 1 se reconstruye el cronograma considerando la vigencia de los ARSAT 1 y 2, los periodos de construcción previstos, los retrasos que se han hecho efectivos a junio del 2020 en cuatro de las construcciones previstas, (a esa fecha no se había iniciado la construcción ni del ARSAT 3, ni del químico 2, ni del híbrido 1, ni del híbrido 2), las especificaciones sobre si los ingresos derivarían de la venta de servicios o la venta del satélite y las potenciales POG en donde se ubicaría cada uno de los satélites.

En el anexo de la ley se presenta el costo estimado de cada satélite dependiendo de la plataforma, y se estima además que los correspondientes a la plataforma eléctrica rondarán los USD 300 millones $^{17}$. Si bien cada plataforma detalla con qué lanzadores tendría compatibilidad, no está determinado qué empresa realizará los lanzamientos. Solo se conoció que para el satélite ARSAT 3, cuya construcción aun no comienza, y cuyo lanzamiento había sido planificado para el 2018, existía un contrato con Arianespace para que se encargara de su lanzamiento. En cuanto al financiamiento de las actividades de investigación, en la ley se

Tabla 1.

Cronograma de fabricación de satélites geoestacionarios de telecomunicaciones

\begin{tabular}{|c|c|c|c|c|c|c|}
\hline Satélite & Plataforma & Fabricación & Lanzamiento & Retraso al 06/2020** & Ingresos por venta & POG \\
\hline ARSAT 1 & ARSAT-3K & $2010-2014$ & 2014 & - & de servicios hasta el 2029 & $71,8^{\circ} 0$ \\
\hline ARSAT 2 & ARSAT-3K & $2012-2015$ & 2015 & - & de servicios hasta el 2030 & $81^{\circ} 0$ \\
\hline ARSAT 3 & ARSAT-3K & 2016-2018 & 2018 & 4,5 años & de servicios hasta el 2033 & $* * *$ \\
\hline Químico 2 & ARSAT $-3 K^{*}$ & 2017-2019 & 2019 & 3,5 años & del satélite hasta el 2021 & - \\
\hline Híbrido 1 & ARSAT- $3 \mathrm{H}^{*}$ & 2016-2019 & 2019 & 4,5 años & de servicios hasta el 2034 & $* * *$ \\
\hline Híbrido 2 & ARSAT- $3 \mathrm{H}^{*}$ & $2019-2022$ & 2022 & 1,5 años & de servicios hasta el 2037 & $* * *$ \\
\hline Híbrido 3 & ARSAT- $3 \mathrm{H}^{*}$ & $2021-2023$ & 2023 & - & de servicios hasta el 2038 & *** \\
\hline Híbrido 4 & ARSAT- $3 \mathrm{H}^{*}$ & $2024-2026$ & 2026 & - & del satélite hasta el 2028 & - \\
\hline ARSAT 1 (Remplazo) & ARSAT-3E & $2026-2029$ & 2029 & - & de servicios hasta el 2046 & $71,8^{\circ} 0$ \\
\hline ARSAT 2 (Remplazo) & ARSAT-3E & $2027-2030$ & 2030 & - & de servicios hasta el 2047 & $81^{\circ} 0$ \\
\hline
\end{tabular}

Notas: * Podrán ser de propulsión química, híbrida o eléctrica, según oportunidad y conveniencia.

** Dado que el plan no estipula un mes para el comienzo de la fabricación, se calculó el retraso desde el primer mes del año en el cual se proyectó dicho inicio.

*** Estos satélites serían puestos en las posiciones actuales y las solicitadas. En caso de que no sean otorgadas, es posible realizar un nuevo proceso de coordinación, operación y ubicarlos en las posiciones orbitales existentes.

Fuente: elaboración propia con base al anexo de la Ley n. ${ }^{\circ} 27.208$.

17 Según personal de la empresa, entrevistado en el 2018, INVAP estaba en ese momento en condiciones de desarrollar una plataforma de propulsión eléctrica, fabricar un satélite de prestaciones equivalentes a las de los ARSAT 1 y 2 , asegurarlo y ponerlo en órbita por un costo total estimado de aproximadamente USD 180 millones incluyendo el desarrollo, que rondaría los USD 40 millones. 
prevé que el mismo esté a cargo del Estado, cuestión que no se está haciendo efectiva al menos en cuanto al desarrollo de la plataforma eléctrica. Respecto a esto, INVAP dio a conocer la creación de la empresa GSATCOM Space Technologies, en conjunto con Turkish Aerospace, un proyecto que tiene como objetivo el desarrollo de una nueva serie de satélites livianos de alto rendimiento para telecomunicaciones, con propulsión full electric. Los satélites tendrán un peso de lanzamiento de entre 1.000 y $2.000 \mathrm{~kg}$, arquitectura HTs, diseño modular escalable que permite customizar el satélite de acuerdo a las necesidades de la misión a menor costo, carga útil flexible y capacidad multilanzamiento, lo cual permite maximizar la utilización de recursos en el lanzamiento ${ }^{18}$. Volviendo al programa de fabricación de satélites, lo único que se informa sobre el financiamiento es que este será con recursos generados por el propio negocio y fondos de organismos de crédito nacionales e internacionales.

Algunos puntos centrales del plan de negocio son los siguientes: el plan no incluye los gastos de desarrollo de las nuevas tecnologías y asume las inversiones recurrentes de IOS ARSAT 1 y 2 como hechas en el primer periodo. Luego de presentar el flujo de inversiones por año, se dedica a hacer los supuestos para las ventas, planteando un ciclo de llenado de la capacidad de los satélites y luego, estimando un precio por MHz y por mbps según la banda para el período 2015-2035. A partir del análisis del mercado mundial se afirma que la demanda está definida por la oferta, de manera que se termina aprovechando su capacidad plena. A la luz de la experiencia con el ARSAT 2, cuya capacidad operativa eficiente demoró más de un año en colmarse, estos supuestos, que hacen a la viabilidad del plan de negocios, dependen de que previo al lanzamiento de los nuevos satélites se desarrolle un efectivo plan de preventa, ya que no necesariamente contar con oferta implica conseguir una demanda efectiva.

Otro punto que se destaca del programa de construcción de satélites es la especificación de la tecnología a utilizar a 20 años vista en un sector sumamente

18 Para más información puede revisar la web de Gsatcom, referenciada al final de este documento. innovador. Para ilustrar esto, alcanza con recordar que la propulsión full electric en satélites geoestacionarios de telecomunicaciones se usó por primera vez $z^{19}$ ocho meses y medio antes de que se sancionara la ley, y que según la consultora Northern Sky Research (NSR), el $25 \%$ de los 106 satélites encargados a nivel mundial por operadores satelitales entre el 2012 y el 2016 incorporaron propulsión eléctrica ${ }^{20}$, ya sea en satélites híbridos (6) o full electric (20). Por sobre esto, es al menos riesgoso basar el plan de construcción de satélites en un plan de negocios que considera proyecciones de precios a 20 años vista en un contexto caracterizado por movimientos constantes tanto en la demanda (e.g. el incremento de la demanda de servicios de telecomunicación satelital móvil para aviones) como en la oferta, en parte debido al auge de las constelaciones de satélites de telecomunicaciones de órbita baja (Wekerle et al., 2017). Si bien los precios no son públicos debido a la confidencialidad de los contratos entre el operador y los proveedores de servicios de información, según referentes de la industria entrevistados, el precio del MHz de capacidad satelital en la Argentina cayó a la mitad entre el 2015 y el 2019, en línea con la tendencia mundial, lo que se explicaría por un aumento sustantivo de la oferta de capacidad satelital. En conclusión, la emergencia de nueva tecnología -algo esperable en un lapso de 20 años en este sector-, podría alterar significativamente tanto la conveniencia técnica como la viabilidad económica del plan de construcción de satélites estipulado en la ley. En este punto resulta importante que ARSAT revise periódicamente el plan en pos de corregir estas divergencias.

En cuanto a la operación de los satélites, en el plan de negocios se aclara que para la banda Ka ARSAT solo comercializará como mayorista, tercerizando la operación por el $25 \%$ de los ingresos a otros comercializadores. Sin embargo, no se establecen cláusulas que determinen la obligatoriedad de que ARSAT sea quien comercialice los servicios de los satélites que eventualmente no sean en banda Ka.

19 El $1 .^{\circ}$ de marzo del 2015 la firma norteamericana Boeing inauguró el primer satélite full electric con el lanzamiento de los GEO ABS-3A y Eutelsat 115 West B en un solo lanzador Falcon 9 de SpaceX. 20 Ver la referencia Henry (2017) en el listado final. 
Respecto a la demora (a junio del 2020) de 4,5 años en el inicio de la construcción del ARSAT 3, en el 2017 se difundió una carta de intención ${ }^{21}$ entre ARSAT y la firma Hughes que despertó controversia en torno a su legalidad e impacto en el futuro desarrollo de la industria satelital argentina. Hughes Network Systems LLC es una firma norteamericana líder mundial en soluciones satelitales y servicios de banda ancha. La carta de intención con Hughes planteaba la creación de una nueva empresa llamada Newco para que lleve a cabo la construcción del ARSAT 3 y lo opere, con una participación accionaria de al menos $51 \%$ para la empresa norteamericana. Al margen de la discusión sobre su legalidad, que no será desarrollada aquí, la carta de intención abre el interrogante sobre si la asociación con una empresa extranjera en los términos en los cuales se planteaba con Hughes, sería la mejor alternativa para que el operador nacional avance en su plan de negocios y le dé viabilidad a la construcción del ARSAT 3. Esta es una cuestión por demás sensible, ya que la actividad del operador nacional es un nodo esencial de un sistema complejo e interconectado: la "economía del espacio" de la Argentina (López, Pascuini \& Ramos, 2017). Por este motivo, las acciones que este lleve adelante impactan en los encadenamientos que se producen desde el punto de vista productivo y tecnológico. Más allá de la controversia, el acuerdo nunca se concretó, por lo que el interrogante respecto a la fabricación del ARSAT 3 continúa abierto.

Para finalizar, se debe analizar la factibilidad de que la planificación para la construcción de satélites planteada en la Ley n. ${ }^{\circ} 27.208$ efectivamente se pueda materializar, y en tal caso, se considere la necesidad de incorporar modificaciones que incluyan criterios acordes a: (i) la verdadera disponibilidad de recursos para que el plan sea llevado adelante; (ii) los requerimientos tecnológicos de la demanda local y global; (iii) las capacidades actualmente al alcance de la industria espacial argentina; y (iv) criterios de eficiencia industrial y comercial. Sobre este último punto (iv), como se argumenta en López, Pascuini \& Ramos (2017):

21 Véase el documento de Carta Hugues ARSAT.
Dada la escala limitada con la cual ha venido operando el sistema hasta el momento, y el hecho de que [en la fabricación de satélites] el comprador ha sido siempre el Estado (que usualmente tiene mayor tolerancia a problemas de sobrecostos, extensión de plazos, etc.); [...] se requiere avanzar hacia la introducción de mayores elementos de eficiencia en el desempeño de los actores de la industria (p. 51).

Con lo cual, parece ineludible que se revisen los modelos de toma de decisiones, así como las prácticas de gestión, organización y comercialización hoy imperantes.

Por otra parte, no existe un plan de fabricación para satélites no geoestacionarios. Si bien la CONAE cuenta con un Plan Espacial Nacional, la última versión disponible, a julio del 2020, es la actualización realizada en el 2010 del Plan para el período 2004-2015. En dicha versión, por ejemplo, los lanzamientos de los satélites SAOCOM 1A у 1B estaban proyectados para el 2014 y el 2015 respectivamente ${ }^{22}$. Como se ha mencionado, el SAOCOM 1A fue lanzado en el 2018, y el 1B en agosto del 2020. Adicionalmente, al menos desde mayo del 2019 en el sitio web oficial de la CONAE figura que la versión preliminar del "Plan Espacial Nacional 2016-2027" ya había sido aprobada por el Directorio de la CONAE y presentada al Poder Ejecutivo. No obstante, a julio del 2020 dicho plan aún no ha sido publicado. En este marco, la actualización y publicación del Plan Espacial Nacional resulta imperante para garantizar la transparencia en el cumplimiento de sus objetivos.

\section{Conclusiones}

La Argentina es parte del selecto grupo de países que pueden desarrollar sus propias tecnologías en la industria satelital, de hecho, es miembro de un grupo aún más selecto que puede diseñar y producir satélites

22 Se puede acceder a la actualización del 2010 en el siguiente link: http://www.cetam.fadu.uba.ar/wp-content/uploads/2015/01/Actualiza cion-Plan-Espacial-2010-2015.pdf 
GEO de telecomunicaciones. Esta es una de las pocas áreas de alta tecnología en la que la Argentina tiene capacidades reconocidas internacionalmente, siendo resultado de un proceso de aprendizaje y desarrollo de capacidades llevado adelante a lo largo de varias décadas. Cuando la estrategia comercial es bien gestionada, caso específico de los satélites geoestacionarios de telecomunicaciones, es una actividad en la que las inversiones luego generan retornos económicos concretos directos e indirectos por la venta de servicios satelitales. Es la actividad que mayores ingresos genera en la industria satelital (según últimos datos públicos relevados para el 2018).

A la luz de estas reflexiones, la indefinición respecto de la continuidad del plan de lanzamiento de satélites de telecomunicaciones, es un factor pone en peligro la continuidad del proceso de aprendizaje y desarrollo tecnológico iniciado años atrás en este sector. Se debe analizar la factibilidad de que la planificación para la construcción de satélites planteada en la Ley $n .^{\circ} 27.208$ efectivamente se pueda materializar, y en tal caso, se considere la necesidad de incorporar modificaciones que incluyan criterios acordes a: (i) la verdadera disponibilidad de recursos para que el plan sea llevado adelante; (ii) los requerimientos tecnológicos de la demanda local y global; (iii) las capacidades actualmente al alcance de la industria espacial argentina; y (iv) criterios de eficiencia industrial y comercial. Sobre este último punto (iv), como se argumenta en López, Pascuini \& Ramos (2017), siendo que el sector satelital en la Argentina ha tenido hasta ahora como principal demandante de satélites locales al Estado, que generalmente es más flexible respecto de los plazos y costos, y ha funcionado a una escala reducida, es necesario que los actores del sector incorporen mayores niveles de eficiencia, y modelos de gestión y organización orientados a la comercialización de los productos locales en el mercado internacional.

La actualización del plan para la construcción de satélites debería contemplar que no será posible desarrollar una plataforma que alcance la eficiencia establecida para los plazos proyectados, en tanto este objetivo debía cumplirse para el 2020, pero a julio del 2020 no se han implementado planes para su realización. También se debería incorporar el hecho de que la demanda de capacidad para los satélites proyectados no está garantizada, y consecuentemente, incorporar este aspecto vinculado a la comercialización en el plan de negocios. Respecto a este último, se deberá tomar nota de la celeridad con la que cambia la tecnología en un sector sumamente dinámico, por lo que la emergencia de nueva tecnología podría alterar significativamente la viabilidad del plan, y los riesgos asociados a asumir precios y demanda a largo plazo.

En términos más generales, esta reflexión suscita a cuestionarse hasta qué punto tiene sentido establecer en una ley y cuál será la tecnología a utilizar a quince años, vista en un sector caracterizado por una fuerte dinámica innovadora. Esto no significa no planificar, sino incorporar mecanismos de flexibilidad y adaptación ante la emergencia de cambios tecnológicos. Es por eso que resulta importante que ARSAT revise periódicamente el plan, algo que, habiendo vencido el primer plazo estipulado de tres años desde la promulgación de la ley, aún no ha sucedido, o al menos no ha sido de conocimiento público.

En este momento la Argentina podría estar brindando servicios desde POG $91,5^{\circ}$ Oeste en bandas Ku y $\mathrm{Ka} ; 38,5^{\circ}$ Oeste en bandas Ku y Ka ; $81^{\circ}$ Oeste en banda Ka; y $71,8^{\circ}$ Oeste en banda Ka, que no solo no se están utilizando, sino que no se han implementado planes para ocuparlas. No utilizarlas implica subutilizar un recurso valioso y escaso como las POG. Si alguna posición no pudiera ser ocupada por un satélite fabricado localmente antes de su vencimiento, existen alternativas que deberían evaluarse a la luz de sus posibles impactos sistémicos, a saber: la subasta o concurso de la POG, la operación de un satélite alquilado, o un modelo hosted payload. La indefinición respecto de la ocupación de estas POG constituye el riesgo de perder un recurso valioso y escaso para la Argentina, en tanto los plazos para colocar un satélite en cada una de ellas vencen en el 2021, 2023 y 2024.

Resulta necesario abordar la cuestión de la organización institucional del sector. En un país con una industria satelital pequeña e incipiente, resulta quizás ineficiente que exista más de un organismo con competencias en la definición de políticas sobre el tema. 
Hasta el momento, ninguno de los actores del Estado involucrados en el sector ha cumplido el rol de definir una visión amplia y estratégica (por ejemplo, el Plan Espacial Nacional 2004-2015, vigente a julio del 2020, no incluye a los satélites de telecomunicaciones). En consecuencia, una primera sugerencia sería que los actores centrales del sistema asociado a la "economía del espacio" en la Argentina se sienten, en coordinación establecida al máximo nivel político, a repensar la división de tareas y responsabilidades, así como un nuevo esquema de gobierno del sector. No se trata exclusivamente de ARSAT y la CONAE, sino también de la entidad encargada de gestionar POG ante la UIT, la de autorizar derechos de aterrizaje, la de sancionar programas de construcción de satélites, el sector privado, etcétera. Esto es importante, en especial de cara a que los distintos campos de aplicación, ya sean de observación, con fines científicos o de telecomunicaciones, sean concebidos bajo una mirada común que abarque además el rol, potencialmente creciente, de los agentes privados en algunos de esos segmentos (López \& Pascuini, 2018).

\section{Agradecimiento}

Se agradece la eficaz colaboración de Valentín Alvarez. Como es usual, cualquier error u omisión en el texto es responsabilidad del autor.

\section{Referencias bibliográficas}

Anselmo, L., \& Pardini, C. (2019). Dimensional and Scale Analysis Applied to the Preliminary Assessment of the Environment Criticality of Large Constellations in LEO. Acta Astronautica, 158, 121-128. https://doi.org/10.1016/ j.actaastro.2017.09.028

Benavídez. (2015, septiembre). Plan satelital geoestacionario argentino 2015-2035. ARSAT. http://servicios.infoleg. gob.ar/infolegInternet/anexos/250000-254999/254823/ ley27208.pdf
Chaari, L., Fourati, M., \& Rezgui, J. (2019). Heterogeneous LoRawAN \& LEo Satellites Networks Concepts, Architectures and Future directions [conferencia]. 2019 Global Information Infrastructure and Networking Symposium (GIIS). https://doi.org/10.1109/giis48668.2019.9044966

El Destape (s. f.). ARSAT: Argentina perdió una nueva posición orbital. El Destape. https://es.scribd.com/docu ment/355202081/ARSAT-Argentina-perdio-una-nuevaposicion-orbital

Gallicchio, N. (2017). Radar and Satellite History. En N. Gallicchio \& K. Teague (Eds), The Evolution of Meteorology: A Look into the Past, Present, and Future of Weather Forecasting (pp. 43-49). https://doi.org/10.1002/978111 9136170.ch5PDFPDF

GSATCOM. (2020). Full Electric HTS Satellite. Flexible Payload and Multi Launch Capability.

Henry, C. (2017, 22 de agosto). All-electric Satellites Halfway to Becoming Half of All Satellites. SpaceNews. https:// spacenews.com/all-electric-satellites-halfway-to-becoming-half-of-all-satellites/

Hughes Network Systems. (2017). Carta Hugues ARSAT. http:// www.unsam.edu.ar/tss/wp-content/uploads/2017/07/ Carta-Hugues-ARSAT.pdf

International Telecommunication Union [ITU]. (2020). Reglamento de Radiocomunicaciones. Conferencia Mundial de Radiocomunicaciones CMR-2020. https://www.itu.int/ pub/R-REG-RR/es

Jakhu, R. (2007). Legal Issues of Satellite Telecommunications, The Geostationary Orbit, and Space Debris. Astropolitics, 5(2), 173-208. https://doi.org/10.1080/1477762 0701580828

Lee, S., Wu, Y., \& Mortari, D. (2016). Satellite Constellation Design for Telecommunication in Antarctica. International Journal of Satellite Communications and Networking, 34(6), 725-737. https://doi.org/10.1002/sat.1128

López, A., \& Pascuini, P. (2018). Institucionalidad y cambio tecnológico en las telecomunicaciones satelitales argentinas. Serie de documentos de trabajo del IIEP, (30), 3-42.

López, A., Pascuini, P., \& Ramos, A. (2017). Al infinito y más allá: una exploración sobre la economía del espacio en la Argentina. Serie de documentos de trabajo del IIEP, (17), 1-61.

López, A., Pascuini, P., \& Ramos, A. (2018). Climbing the Space Technology Ladder in the South: The Case of Argentina. Space Policy, 46, 53-63. https://doi.org/10.1016/j. spacepol.2018.06.001

López, A., Ramos, A., \& Pascuini, P. (2019). Economía del espacio y desarrollo: el caso argentino. CTS: Revista Iberoamericana de Ciencia, Tecnología y Sociedad, 14(40), 11-133. 
Nagendra, N., \& Basu, P. (2016). Demystifying Space Business in India and Issues for the Development of a Globally Competitive Private Space Industry. Space Policy, 36, 1-11. https://doi.org/10.1016/j.spacepol.2016.02.006

Orvola, A., Nogueira, R., \& Chimenti, P. (2020). The Present and Future of the Space Sector: A Business Ecosystem Approach. Space Policy, 52. https://doi.org/10.1016/j.spa cepol.2020.101374

Pardini, C., \& Anselmo, L. (2020). Environmental Sustainability of Large Satellite Constellations in Low Earth Orbit. Acta Astronautica, 170, 27-36. https://doi.org/10.1016/j. actaastro.2020.01.016

Qu, Z., Zhang, G., Cao, H., \& Xie, J. (2017). Leo Satellite ConsteIlation for Internet of Things. IEEE Access, 5, 18391-18401. https://doi.org/10.1109/ACCESS.2017.2735988

Quiroga, J. (2020). Capacidades dinámicas en la producción de bienes intensivos en conocimientos. El caso del desarrollo de radares en Argentina (2003-2015). CTS - Revista Iberoamericana de Ciencia, Tecnología y Sociedad. https: //doi.org/10.13140/RG.2.2.27266.63680

Quiroga, J., \& Aguiar, D. (2016). Abriendo la "caja negra" del radar. Las políticas de radarización para uso civil y de defensa en Argentina entre 1948 y 2004. H-industri@, 10(19), 71-100. https://ri.conicet.gov.ar/handle/11336/66875
SIA (s.f.). State of the Satellite Industry Report. https://sia.org/ news-resources/state-of-the-satellite-industry-report/

Solis-Santomé, A., \& Santos-Reyes, J. (2016). Diagnóstico de la situación actual de los satélites. Humanidades, Tecnología y Ciencia del Instituto Politécnico Nacional, 14, 1-15.

Urbina, J. (2017). El espacio, futuro de la fuerza aérea colombiana. Ciencia y Poder Aéreo, 12(1), 202-208. https://doi. org/10.18667/cienciaypoderaereo.572

Wekerle, T., Pessoa, J., Costa, L., \& Trabasso, L. (2017). Status and Trends of Smallsats and their Launch Vehicles - An Up-to-date Review. Journal of Aerospace Technology and Management, 9(3), 269-286. http://dx.doi.org/10.5028/ jatm.v9i3.853

Wood, D., \& Weigel, A. (2012). Charting the Evolution of SateIlite Programs in Developing Countries - The Space Technology Ladder. Space Policy, 28(1), 15-24. https://doi. org/10.1016/j.spacepol.2011.11.001

Xu, J., \& Zhang, G. (2018). Design and Transmission Performance Analysis of Satellite Constellation for Broadband LEO Constellation Satellite Communication System Based On High Elevation Angle. IOP Conference Series: Materials Science and Engineering, 452(4), 042092. https://doi.org/1 0.1088/1757-899X/452/4/042092 